\title{
Recomendaciones de la Sociedad Uruguaya de Medicina Intensiva sobre los aspectos bioéticos en la pandemia Covid-19
}

Recommendations by the Uruguayan Society of Intensive Care on the Covid-19 pandemic Recomendações da Sociedade Uruguaia de Medicina Intensiva sobre aspectos bioéticos na pandemia Covid-19

Este es un documento oficial de la Sociedad Uruguaya de Medicina Intensiva elaborado por la Comisión de Bioética

Dres. Álvaro Giordano ${ }^{1}$, Ana Canale², Julio Pontet ${ }^{3}$, Natacha Reyes ${ }^{4}$, Armando Cacciatori' Humberto Correa ${ }^{6}$, Luis Andrés Núñez

\section{Resumen}

Introducción: la pandemia provocada por el SARS-CoV-2 genera un importante desafío para el sistema sanitario y especialmente para la Medicina Intensiva. Es necesario prepararse en múltiples aspectos. Además, considerar plausible una demanda extraordinaria de camas críticas que puede llevar a un desbalance entre las necesidades clínicas y la disponibilidad efectiva de los recursos sanitarios.

Objetivos: realizar un análisis bioético para brindar una orientación en la atención a los pacientes críticos. Objetivos específicos: 1) Analizar los principios bioéticos fundamentales en este contexto. 2) Apoyar a los clínicos en la toma de decisiones difíciles. 3) Hacer explícitos los criterios de asignación de recursos. 4) Definir líneas de acción ante un posible escenario de "desastre sanitario".

Método: la SUMI ha generado un ámbito de trabajo colectivo cuyo método de trabajo fue la deliberación. En la documentación se utiliza la revisión bibliográfica y los protocolos ya existentes.

Resultados: el trabajo plantea un análisis teórico documentado sobre los principios bioéticos involucrados en el contexto de pandemia, sobre los escenarios de demanda asistencial y sobre la fundamentación para un cambio en los criterios éticos ante un escenario de saturación del sistema.

Conclusión: se plantean recomendaciones prácticas para: 1) Toma de decisiones de ingreso y egreso en demanda controlada. 2) Criterios de acción ante el aumento de la demanda estableciendo definiciones de los diferentes escenarios. 3) Recomendaciones para aplicar en un escenario de saturación del sistema.

\footnotetext{
1. Médico intensivista. Mg. en Bioética. Coordinador UMI Hospital Español, ASSE. Director CTI de COMEF.

2. Médico intensivista. Coordinadora UCI Hospital Pasteur, ASSE.

3. Médico intensivista. PhD. Director UCl Hospital Pasteur. Especialista en Administración y Gestión en Salud.

4. Médico intensivista y paliativista.

5. Médico intensivista. Profesor Adjunto del Sector Procuración del Instituto Nacional de Donación y Trasplante (INDT). Universidad de la República. Ex Jefe del Centro de Cuidados Especiales (CCE) del Hospital Central de las Fuerzas Armadas. Ex Asistente del Servicio de Medicina Intensiva del Centro Nacional de Quemados (CE.NA.QUE). Universidad de la República. Especialista en Gestión de Servicios de Salud. Universidad CLAEH.

6. Médico intensivista. Ex Profesor de Medicina Intensiva. Facultad de Medicina, Universidad de la República. Decano emérito de la Facultad de Medicina de la Universidad CLAEH. Profesor de Humanismo Médico de la Facultad de Medicina de la Universidad CLAEH. Miembro (Correspondiente) de la Academia Nacional de Medicina.

7. Médico intensivista. Magíster en Gestión de Empresas de Salud. Ex Asistente de la Cátedra de Medicina Intensiva. Hospital de Clínicas, Universidad de la República. Presidente de la Sociedad Uruguaya de Medicina Intensiva (2018-2020).

Los autores declaran no tener conflictos de intereses.
}

Recibido: $20 / 5 / 20$

Aprobado: 21/10/20

Attribution-NonCommercial 4.0 International (CC BY-NC 4.0) 
Palabras clave: Pandemias

Covid-19

Bioética

Cuidados críticos

$\begin{array}{ll}\text { Key words: } & \text { Pandemic } \\ & \text { Covid-19 } \\ & \text { Bioethics } \\ & \text { Critical care }\end{array}$

por Covid-19 o cualquier otra emergencia sanitaria que enfrente el sistema de salud. Si bien está dirigido a la Medicina Intensiva, sus conceptos son aplicables a otras áreas de la asistencia pública, y consideramos fundamental el aporte de todos los colegas que toman pacientes graves a su cargo.

La incertidumbre está omnipresente en todo acto médico y por lo tanto en la relación médico/paciente, implícita o explícitamente, aunque esta no necesariamente genere un dilema consciente. A mayor incertidumbre y cuanto más explícita es para el profesional, surgen los problemas éticos acerca de cómo "hacer lo correcto" en la práctica clínica, principalmente cuando los deberes o valores comienzan a entrar en conflicto.

Gestionar la incertidumbre implica planificar los dilemas éticos previsibles, identificando las decisiones que pueden generar mayor conflicto, utilizando o creando herramientas y mejorando los procesos de estas decisiones.

\section{Objetivos específicos}

1. Analizar los principios bioéticos fundamentales que involucran a la Medicina Intensiva, su aplicación en la práctica asistencial, y su enfoque en el contexto de la pandemia por Covid-19.

2. Apoyar a los clínicos en la toma de decisiones difíciles, que conllevan un estrés emocional al enfrentar la responsabilidad en la "selección de pacientes".

3. Hacer explícitos los criterios de asignación de recursos de salud en una situación extraordinaria ante el personal sanitario y ante toda la sociedad.

4. Definir líneas de acción dirigidas a prevenir y a actuar en un posible escenario de "catástrofe o desastre sanitario", entendiendo por tal aquel en el que el sistema no pueda cubrir las demandas asistenciales.

\section{Los principios bioéticos involucrados en la Medicina Intensiva y el desafí́ ético en la pandemia}

El principal principio ético que nuestra sociedad acepta para el trabajo en salud y que inspira las últimas normativas legales es el respeto a la dignidad de las personas ${ }^{(1,2)}$. El término dignidad es de difícil definición, pero en este contexto adquiere especial relevancia. Más allá de todas las consideraciones que se harán, nunca debemos olvidar el valor intrínseco de la vida humana durante toda su existencia y el respeto a los derechos humanos 
de todas las personas. La importancia de este principio no puede modificarse por las circunstancias.

A nivel de la asistencia sanitaria los principios tradicionales de la bioética ${ }^{(3)}$ tienen un rol preponderante en el trabajo normal de la Medicina Intensiva.

- El principio de Beneficencia. Hacer el bien y obrar por bien del paciente ${ }^{(4)}$. Es un principio aceptado por todos los niveles sociales, por el equipo de salud y usuarios del sistema que ha sido la base de la medicina tradicional.

- El principio de No maleficencia que pone un límite al denodado esfuerzo por hacer. Resulta un principio clave ante las enormes posibilidades técnicas de hacer, y lo primero es no hacer daño: primun non nocere. En la Medicina Intensiva los conceptos de adecuación del esfuerzo terapéutico, la proporcionalidad y la futilidad, se basan en buena parte en este principio. Tiene también un rol fundamental en el contexto de la investigación y búsqueda de fármacos contra el SARS-CoV-2.

- El principio de Autonomía, que emana del concepto de libertad en todas las circunstancias vitales, tiene un creciente reconocimiento por la población, quizás no con la prioridad que tiene en el mundo anglosajón. Los derechos del hombre y del ciudadano, que eran derechos públicos, han avanzado hacia la vida privada de las personas en el estado liberal. $\mathrm{La}$ asistencia a la salud debe razonar con un doble molde en que importa lo que es bueno para la persona, pero es ella quien debe decir qué es lo bueno ${ }^{(5)}$.

- El principio de Justicia. Entendido como la justicia distributiva en que la sociedad debe destinar recursos en forma equitativa cuando de salud se trata. $\mathrm{La}$ justicia distributiva es un marco general que condiciona todas las decisiones de salud y en el que a veces no somos conscientes. Allí está la posibilidad de realizar una resonancia o una angiografía, indicar un fármaco u otro, una técnica u otra. Se torna especialmente relevante en el contexto de demanda extraordinaria de recursos.

Más allá de estos principios, la situación actual impone la consideración de otros principios y valores que deben estar presentes en el contexto de la pandemia Covid-19 y ante una posible demanda extraordinaria de recursos.

Deber moral de tratar: este deber, resaltado en diversas publicaciones como duty to treat (el deber de tratar), nos obliga a tratar a los enfermos postergando necesidades personales, hecho que se hace a veces dramáticamente real en situaciones de desastres ${ }^{(6,7)}$. La Medicina Intensiva en su conjunto, todos sus recursos humanos, desde los licenciados de enfermería a los auxiliares de servicio, desde los enfermeros a los jefes de servicio, siempre ha dado respuesta a estas obligaciones con una riquísima historia de esfuerzos y postergaciones personales. Anotar este deber que ha sido tantas veces cumplido, es también un reconocimiento a la rica historia de los trabajadores de la Medicina Intensiva que siempre han estado a la altura.

Deber de autocuidado. La Constitución de la República en su artículo 44 señala "Todos los habitantes tienen el deber de cuidar su salud, así como el de asistirse en caso de enfermedad...". Además, se debe destacar el valor instrumental de todos los trabajadores de la salud, de modo que el cuidado personal es un deber personal y para con los otros trabajadores a fin de evitar el contagio, y para con la sociedad que cuenta con un recurso calificado y escaso.

Principio de protección del personal sanitario. Se debe garantizar, por razones éticas de reciprocidad, el cuidado y la protección de los trabajadores de la salud, quienes, en la emergencia sanitaria, asumen mayores riesgos por cumplir con el deber de cuidar a la población.

Deber de planificación sanitaria. La planificación en situaciones de escasez de recursos es prioritaria. Si no se realiza, puede llevar a la gestión inapropiada de la crisis, al desperdicio de recursos, a la pérdida inadvertida de vidas, a la pérdida de confianza en el sistema sanitario y a decisiones innecesarias de triaje/racionamiento. La planificación proactiva es el primer eslabón de la cadena para reducir la morbilidad, la mortalidad y otros efectos indeseables de un desastre emergente ${ }^{(8)}$.

Deber de respuesta de las instituciones de salud. Las instituciones sanitarias tienen el deber social de responder de manera eficiente a las nuevas necesidades, en condiciones de apremios económicos como se ha observado en esta circunstancia.

\section{Definición de escenarios}

Definir escenarios es de fundamental importancia para el enfoque que el sistema de salud tiene que dar al problema de la pandemia por Covid-19.

Desde el punto de vista logístico, el desafío está en disponer de los recursos, principalmente de recursos humanos de calidad. En la actualidad existe un déficit de profesionales de cuidados intensivos (tanto médicos como no médicos), el cual no será posible de solucionar en el corto o mediano plazo.

Desde el punto de vista ético implica una valoración exhaustiva de cada caso a fin de brindar la asistencia al paciente que realmente se beneficie de un tratamiento intensivo, con necesidad de soporte vital.

Ambos desafíos constituyen factores comunes a los dos escenarios que vamos a definir. 


\section{a) Demanda controlada}

Al momento actual de presentar estas recomendaciones (mayo de 2020), este es el escenario en el que nos encontramos, donde la demanda asistencial puede ser absorbida por el sistema de salud.

Lograr esto depende de una serie de políticas sanitarias y extrasanitarias que tiene por objetivo disminuir la cantidad de contagios, evitando los brotes epidémicos, y con ello disminuir la cantidad de personas infectadas que requieran asistencia en los tres niveles de atención en un breve período.

El tercer nivel, que correspondería a los cuidados intensivos, se caracteriza por una rigidez, producto de su complejidad (instalaciones, equipos, profesionales) que hace difícil su expansión. No obstante, se deben realizar los máximos esfuerzos para lograr ampliar la oferta de estos servicios, de forma tal que se puedan absorber la primera serie de casos y posteriormente exista una capacidad que pueda satisfacer la demanda que probablemente se continúe generando.

Por otra parte, debemos recordar que las UCI, con o sin pandemia, deben recibir a pacientes graves con otras patologías, y las mismas medidas tendientes a controlar la pandemia pueden tener efectos no deseados en poblaciones con enfermedades crónicas, que han visto resentidos sus controles en salud y por ende tener un riesgo aumentado a presentar exacerbaciones graves.

\section{b) Saturación del sistema}

Es un escenario hipotético no deseado, pero probable, el cual no podemos ni debemos descartar. Tenemos el deber moral de prepararnos lo mejor posible.

Se define cuando la oferta de servicios de cuidados intensivos se ve superada por la demanda y se han realizado los máximos esfuerzos posibles para aumentar la disponibilidad de los mismos (camas UCI y ventiladores), así como haber evaluado la posibilidad de transferencia de pacientes a centros con mayor disponibilidad de dichos recursos.

En este escenario de medicina de catástrofe es necesario un racionamiento de recursos con un criterio de justicia distributiva $^{(7)}$.

A su vez, puede ser necesario que la asistencia a pacientes críticos se realice en otras instalaciones hospitalarias, como salas de recuperación anestésica, block quirúrgico, emergencia e incluso salas de cuidados moderados, que no fueron creadas para tal fin y cuyo personal no está debidamente entrenado en el manejo de estos pacientes. La realidad es la que impone este tipo de trabajo, sabiendo que no es lo ideal.

\section{c) Condiciones que determinan el cambio de escenario}

Es difícil determinar un punto exacto donde termina el escenario de demanda controlada y se pasa a uno de saturación.
Resulta claro que el elemento a tener en cuenta es el recurso UCI, y, por lo tanto, más que definir un número exacto de camas es necesario primero agotar otras estrategias y definir un umbral.

Entendemos que para definir este umbral es necesario:

1. Un conocimiento y monitoreo en tiempo real de la capacidad de brindar cuidados intensivos en el país con las estructuras apropiadas y con el personal adecuado.

2. Las diferentes UCI deben funcionar como un sistema integrado en el cual se puedan repartir las cargas de trabajo con la finalidad de lograr una mayor eficiencia, disminuyendo la posibilidad de que pacientes no puedan acceder a este recurso porque el mismo no se encuentre disponible en su comunidad habiendo capacidad ociosa en otros.

3. Mantener siempre una fluida comunicación entre las UCI y otros servicios, destacándose las salas de cuidados moderados en las cuales debe existir siempre capacidad de recibir los egresos de las UCI.

4. Umbral de ocupación: los umbrales deben servir no solo de alerta, sino también de catalizadores para determinadas acciones. El nivel de ocupación que determina la alerta es del $85 \%$ de la máxima capacidad instalada, sumado a la imposibilidad de otorgar altas en el corto plazo (12 horas). No consideramos prudente llegar a un 100\% de la ocupación para emitir la alerta al sistema, ya que en esa situación es probable que no podamos dar asistencia a otro paciente, ya estaríamos en un escenario de saturación.

5. Una vez que se emite la alerta, el sistema debe asignar una cama en otra institución que disponga del recurso, siempre y cuando se pueda trasladar al enfermo (tomando en cuenta la condición clínica de éste y disponibilidad logística para su traslado).

6. Si no se puede satisfacer la demanda, habiendo agotado todas las posibilidades de ampliar recursos o transferir pacientes a otros centros, estamos en el escenario de saturación del sistema y por ende se deben aplicar criterios de racionamiento.

\section{Recomendaciones. Escenario 1: Demanda controlada}

\section{a) Planificación anticipada}

Los criterios de ingreso a UCI se basan en los principios bioéticos mencionados y tienen en cuenta al paciente en su globalidad y no solo el enfoque de la enfermedad aguda.

El proceso de admisión de un paciente a UCI es dinámico. No obstante, es claro que hay pacientes que no serán ingresados a cuidados intensivos y que esa decisión se puede tomar desde su ingreso a un hospital o incluso 
previamente. En estos casos están los pacientes con muy baja expectativa de sobrevida y también aquellos que no quieren ingresar por expresa voluntad en el ejercicio de su autonomía.

En base a esto, independientemente del escenario en el que se encuentre el sistema, es recomendable identificar a cada paciente que ingresa a un hospital si es o no pasible de recibir cuidados críticos o intensivos, y si lo es, la intensidad y proporcionalidad de dicho tratamiento. En caso de que no sea pasible, se debe garantizar un tratamiento adecuado a las necesidades del paciente, según la situación clínica en que se encuentre, convocando al equipo de cuidados paliativos para su seguimiento de manera de garantizar una mejor calidad de asistencia en esta etapa de la vida (ver anexo 1: Rol de los cuidados paliativos).

Teniendo en cuenta estos preceptos, entendemos que en todo paciente que ingresa a un hospital es necesario:

\section{Valorar}

a. Edad: no solo la cronológica, sino también la biológica.

b. Comorbilidades/Enfermedades de base y estadio de éstas.

c. Severidad de la enfermedad aguda. Utilizando scores como: SOFA (Sequential Organ Failure Assessment), APACHE II (Acute Physiology and Chronic Health disease Classification System II), SAPS II (Simplified Acute Physiology Score II). Se debe tener en cuenta que cualquier score de severidad que se utilice puede subvalorar el riesgo.

\section{Explorar}

a. Deseos y expectativas del paciente o familiar a cargo.

b. Si dispone de voluntades anticipadas, seguir sus instrucciones, dejar constancia en historia clínica de forma rápidamente visible y notificando a los médicos y personal responsable de su cuidado.

Es importante aclarar que la edad cronológica per se no constituye una limitante al ingreso, debe ser integrada a otros elementos de valoración como la presencia de comorbilidad y el grado de fragilidad o vulnerabilidad.

Para la valoración de los puntos a y b recomendamos el uso combinado de herramientas como NecPal CCOMS-ICOC (NECesidades Paliativas del Centro Colaborador de la OMS $)^{(9)}$, marcador del grado de severidad/gravedad de mal pronóstico asociado a escalas que miden el grado de fragilidad (grado de reserva o vulnerabilidad del paciente), como la escala de fragilidad clínica (EFC) de Rockwood ${ }^{(10)}$. Esto permite la toma de decisiones en los momentos de gravedad clínica y conciliar intensidad terapéutica con momento de vida, gravedad de la enfermedad(es) y necesidades del paciente ${ }^{(8,11,12)}$.

Dicho esto, es importante mencionar que el equipo de salud deberá usar aquellas herramientas con las que esté familiarizado tanto para estadificar la enfermedad de base o comorbilidad (NYHA, GOLD, ChildPugh Score, escala de Karnofsky), como para valorar la severidad de la enfermedad aguda (SOFA, APACHE II, SAPS II).

De esta forma, se procurará establecer en todos los pacientes hospitalizados una planificación anticipada mediante una definición clara y visiblemente documentada en la historia clínica de que, en caso de ser necesario, no se beneficiará de reanimación cardiopulmonar (RCP), maniobras invasivas de sostén vital, como intubación oro o nasotraqueal (IOT/INT), ventilación mecánica invasiva (VMI), hemodiálisis, otras, y/o ingreso a UCI, además de la debida notificación al equipo de salud que asistirá a dicho paciente.

\section{b) Criterios de selección/ingreso a UCI}

Se pueden aplicar diferentes criterios a fin de admitir al paciente más indicado para ingresar a una UCI. El ingreso debe ir condicionado de acuerdo a variables de reversibilidad y beneficio, por lo tanto, ingresará a la UCI todo aquel que verdaderamente se beneficie de ella.

El criterio más extendido ${ }^{(8,11,13)}$, y por otro lado conocido y aplicado en nuestro medio, es el modelo de priorización, el cual toma en consideración la enfermedad aguda, pero también la enfermedad de base o comorbilidades y sus posibilidades de revertir.

Es así que se establece un modelo de cuatro prioridades (tabla 1).

- Prioridad 1: pacientes críticamente enfermos, con razonable probabilidad de que su situación revierta si son tratados en UCI. Requieren monitoreo continuo y tratamiento enérgico que no puede ser ofrecido en otra área que no sea la UCI (AVMI, apoyo vasopresor, hemodiálisis, ECMO). No existe una limitación en el esfuerzo terapéutico, tanto en intensidad como en extensión, por lo cual su ingreso es indiscutible.

- Prioridad 2: pacientes inestables que requieren monitorización y eventual intervención inmediata. No requieren AVMI en el momento de la valoración (algunos la pueden requerir en la evolución), pero sí altos requerimientos de oxigenoterapia y/o la presencia de una disfunción. A diferencia de prioridad 1, pueden asociar comorbilidad leve o moderada, que complique su evolución e inclusive la reversibilidad del cuadro actual. Habitualmente ingresan a 
Tabla 1. Criterios de priorización para la admisión de un paciente a UCl en un contexto normal (demanda controlada).

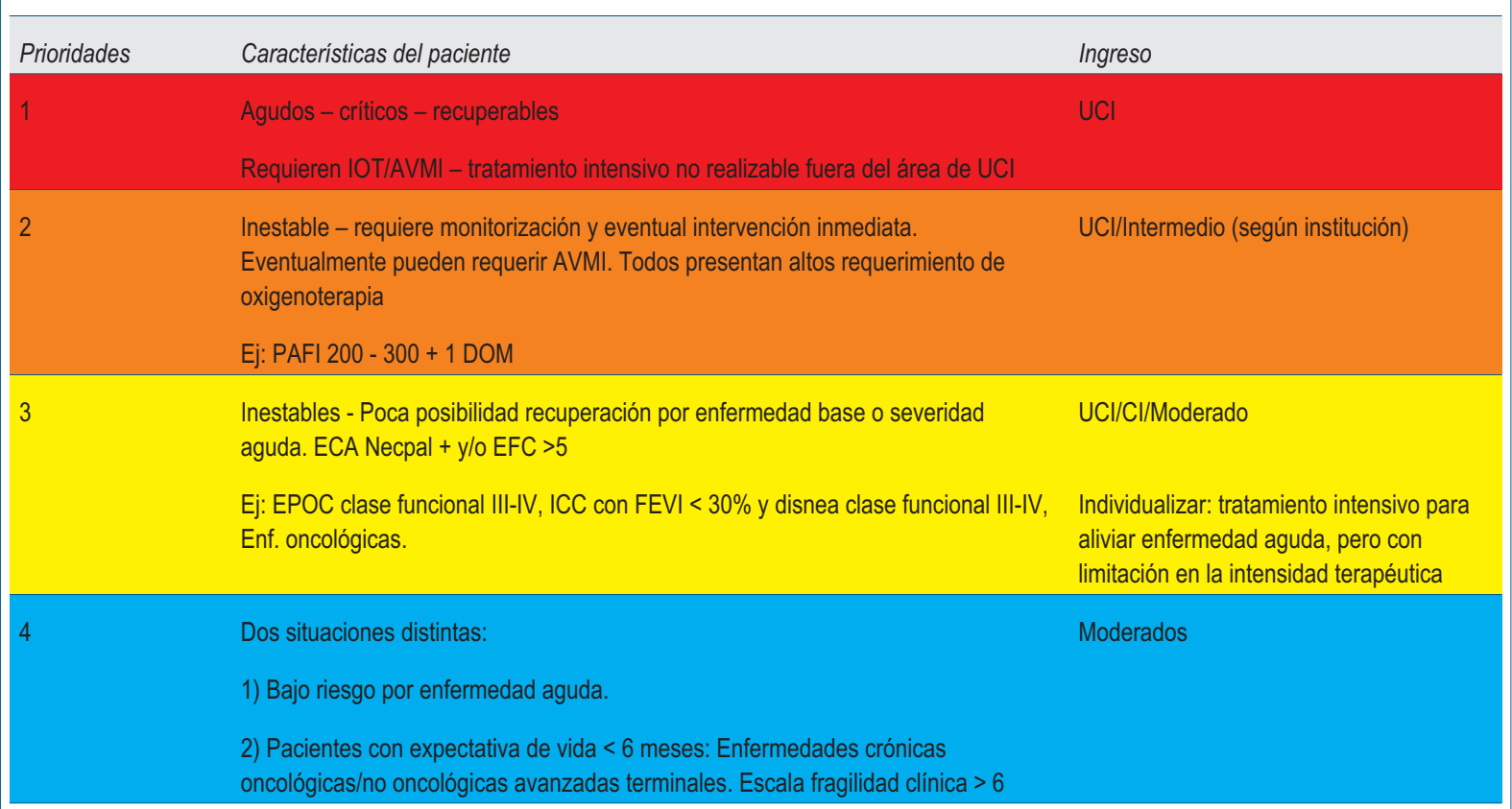

ECA: enfermedad crónica avanzada; IOT: intubación orotraqueal; AVMI: asistencia ventilatoria mecánica invasiva; DOM: disfunción orgánica múltiple.

UCI o unidades de cuidado intermedio dependiendo de la institución en que se encuentren.

- Prioridad 3: el potencial de reversibilidad de su condición actual es menor que para las categorías precedentes, generalmente por la presencia de comorbilidades severas, y por ello el beneficio real de su ingreso a UCI es discutible. Si bien son pasibles de recibir tratamiento intensivo, para intentar revertir la agudización de su enfermedad, en muchos casos es necesario discutir o acordar un límite en la intensidad terapéutica (por ejemplo: no implementar AVMI, no implementar RCPA en caso de PCR). Los ejemplos más típicos son la EPOC estadio Gold III-IV, la ICC con clase funcional III y/o FEVI menor o igual a $30 \%$. Para este tipo de pacientes, las herramientas NecPal y la EFC resultan de gran utilidad al estandarizar la condición del paciente y disminuir los sesgos en el juicio clínico ${ }^{(9,10)}$.

- Prioridad 4: son los pacientes que se encuentran en los dos extremos de la gravedad (demasiado bien o demasiado enfermos) y por lo tanto no se verán beneficiados de ingresar a UCI (por ejemplo: pacientes con enfermedad oncológica estadio IV, pacientes con alto nivel de dependencia).

Este modelo de cuatro prioridades tiene la ventaja de ser flexible, permite adaptar el triaje de acuerdo al balance entre demanda/oferta de recursos en un momento dado y de acuerdo a la realidad de cada institución. Aplicable aun con cambios rápidos en la disponibilidad de recursos (camas UCI, ventiladores), permitiendo a todos los pacientes con necesidad de UCI permanecer elegibles ${ }^{(14)}$.

\section{c) Ingresados en UCI: inicio, revisión y terminación del tratamiento crítico}

Una vez que el paciente ingresa a UCI, es necesario establecer una serie de pautas o criterios, los cuales son válidos independientemente del escenario ${ }^{(8,11,15)}$.

Éstos a su vez deben ser comunicadas en forma clara y transparente al paciente si es posible y/o sus familiares, y asegurarse que los mismos fueron capaces de comprender la información aportada.

1. Establecer un plan terapéutico claro: con objetivos claros y con una revisión diaria de los resultados. Dada la dinámica de estos pacientes, las pautas no pueden ser rígidas, por lo que pueden ir modificándose sobre la marcha.

2. Actuación frente a eventos mayores: desde el ingreso se debe establecer si el paciente será pasible o no de escalamiento en medidas invasivas y eventualmente RCP en el caso de presentar paro cardiorrespiratorio. 
Tabla 2. Criterios de priorización para la admisión de un paciente a UCl en un contexto de saturación

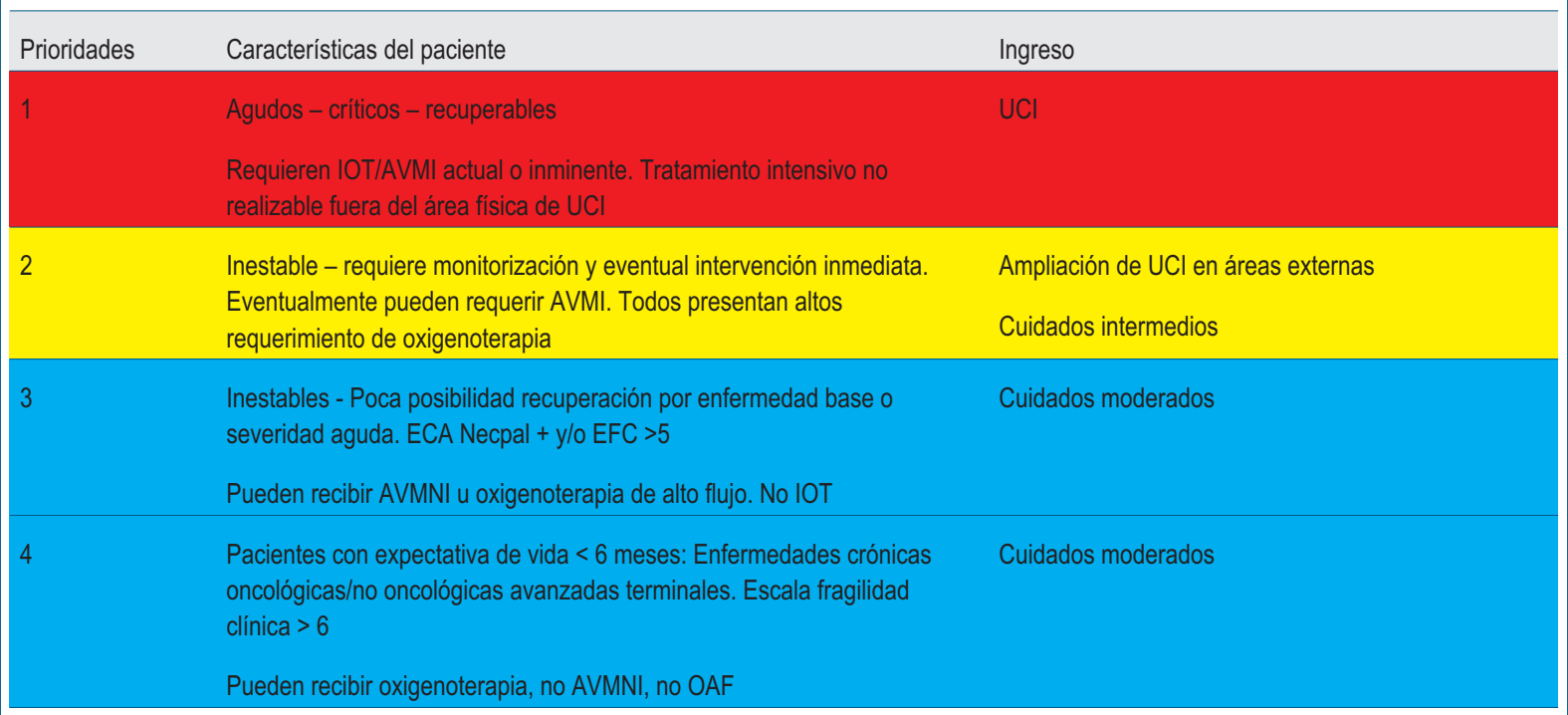

ECA: enfermedad crónica avanzada; IOT: intubación orotraqueal; AVMI: asistencia ventilatoria mecánica invasiva; AVMNI: asistencia ventilatoria mecánica no invasiva; OAF: oxigenoterapia de alto flujo.

3. Desvinculación de la AVMI: agilizar los procedimientos de desvinculación de la AVMI, según medicina basada en la evidencia y así como los protocolos de derivación de los pacientes a sala u otros centros de derivación para liberar recursos para otros pacientes.

4. Prueba de UCI: este concepto se aplica cuando existen dudas en beneficio del tratamiento invasivo. En estos casos se establecen objetivos a conseguir en el corto plazo (primeras 72 horas), que, de no alcanzarse después de sostén vital total, se considerará la futilidad de los mismos y la necesidad de ser retirados y readecuar la terapéutica, priorizando el confort.

5. Readecuación terapéutica: las decisiones respecto a la readecuación en la intensidad terapéutica pueden ser una solicitud explícita del paciente, en uso de su autonomía, o de su familia en caso de no estar éste en condiciones de expresarse, o del equipo asistencial que llega a la conclusión del agotamiento de la estrategia implementada, pero siempre deben ser consensuadas entre las partes. Cuando se decide la retirada de las medidas terapéuticas pretendidamente curativas, se debe actuar sin dilación, aplicando concomitantemente las medidas paliativas que correspondan.

6. Cuidados paliativos: convocar al equipo de cuidados paliativos para procurar una continuidad asistencial en sala de aquellos pacientes que por la irreversibilidad de la situación clínica se suspendieron los tratamientos pretendidamente curativos y se pasó a un cuidado sintomático priorizando el confort (ver anexo Rol de los cuidados paliativos).

\section{d) Criterios de egreso}

Egresarán de la UCI aquellos pacientes que dejen de revestir las condiciones de admisión.

Se puede dar en las siguientes condiciones:

1. Estabilidad fisiológica que no requiera de monitorización y cuidados intensivos, completando su tratamiento y evolución en sala de cuidados moderados.

2. Adecuación terapéutica, retirada de medidas y/o mala evolución, es adecuado favorecer el traslado a un área de menor complejidad.

\section{Fallecimiento.}

En aquellos casos en los que el paciente es egresado a un sector de menor complejidad, se debe coordinar el traslado con el equipo médico del sector al cual será transferido de manera de mantener la continuidad asistencial. De preferencia, y si está disponible, se recomienda un seguimiento por el equipo de Medicina Intensiva extramuros.

En el caso particular actual de pandemia que nos afecta, de tratarse de paciente Covid-19 positivo, se debe seguir el protocolo establecido en la institución de salud para el traslado del paciente y las medidas de aislamiento correspondientes. 


\section{Recomendaciones. Escenario 2: saturación del sistema}

Como mencionamos, en este escenario excepcional, es necesario un racionamiento de recursos con un criterio de justicia distributiva.

\section{a) Fundamentación ética}

Los autores no tienen experiencia en una situación de catástrofe como la que podría ocurrir, con saturación del sistema sanitario. En la bibliografía revisada se coincide en la necesidad de ampliar los principios éticos utilizados en situación normal dando lugar a otros principios éticos que no son los preferidos por la sociedad ni por el cuerpo médico. La selección de los artículos citados, entre varios otros, responde a la proximidad cultural y al conocimiento de los autores. Es importante señalar que se trata de una situación hipotética de catástrofe sanitaria donde no hay una elección libre de ninguno de los actores, sino una imposición de las circunstancias.

El aspecto ético más relevante se halla constituido por el obligado cambio de enfoque de los principios éticos que sostienen la toma de decisiones ${ }^{(16)}$. Es un escenario donde pueden ser necesarios criterios de acceso a cuidados intensivos (y de egreso), no solo estrictamente de adecuación clínica y de proporcionalidad de la atención, sino también en base a un criterio lo más compartido posible de justicia distributiva y de una asignación adecuada de recursos de asistencia sanitaria limita$\mathrm{da}^{(8,15)}$.

De acuerdo con Beca, en un artículo motivado por el terremoto de Chile en 2009, el enfoque debe realizarse desde la ética de la Responsabilidad. En situaciones de desastres, las decisiones necesitan subordinarse a las necesidades de bien común, priorizar la Justicia por sobre la Beneficencia, aun ante casos individuales ${ }^{(7)}$.

Este escenario de saturación puede asimilarse sustancialmente al campo de la "medicina de desastres", para el cual la reflexión ética ha desarrollado con el tiempo muchas indicaciones concretas para los médicos $\mathrm{y}$ enfermeras que toman decisiones difíciles ${ }^{(15)}$.

Un artículo de E. Emanuel, motivado por la situación de pandemia, resume la fundamentación en forma clara $^{(17)}$. Sostiene que hay cuatro valores fundamentales que ya han sido debatidos para incluir y trabajar en el contexto del extraordinario disbalance entre demandas y recursos. 1. Maximizar beneficios. 2. Trato igualitario. 3. Promover y recompensar el valor instrumental. 4. Dar prioridad a los menos favorecidos*.

1. Maximizar los beneficios resulta un objetivo prioritario. Entendido como procurar la mayor supervi-

\footnotetext{
* La explicación de los términos es a modo de resumen y presenta di-
} ficultades de traducción. vencia del colectivo, sea en el logro del mayor número absoluto de personas que logran sobrevivir o en el mayor número de años de vida recuperados, dando prioridad a los pacientes con mayor probabilidad de sobrevivir más tiempo después del tratamiento.

2. El trato igualitario implica que no puede haber discriminación en la asistencia. No son aceptables las diferencias arbitrarias por grupo étnico, sexo, edad, condición social. Incluso algunas diferencias normalmente aceptadas que implican diferencias en la capacidad de pago, no deben ser aceptadas en el contexto de pandemia. Tratar a las personas por igual podría intentarse mediante una selección aleatoria, como una lotería, o por una asignación por orden de llegada.

3. El valor instrumental podría ser promovido dando prioridad a los que pueden salvar a otros, o recompensar dando prioridad a los que salvaron a otros en el pasado.

4. Dar prioridad a los menos favorecidos debe ser entendido como dar prioridad, ya sea a los más enfermos o a los más jóvenes en malas condiciones clínicas, considerando que si no se tratan, habrán tenido vidas más cortas, truncadas por la pandemia ${ }^{(17)}$.

Si bien estas recomendaciones procuran ayudar a los profesionales a la "toma de decisiones", no debemos obviar que se actuará más como gestores de una situación de desastre que como decisores libres, puesto que estas decisiones están absolutamente condicionadas por las circunstancias externas ${ }^{(16)}$.

\section{b) Recomendaciones}

En base a lo expuesto, entendemos que en un escenario de saturación del sistema es necesario realizar las siguientes recomendaciones:

1. Todos los pacientes deben ser asistidos. El derecho a la asistencia en salud es uno de los derechos humanos y no se puede negar la atención a ninguna persona. Esa asistencia será proporcional a las necesidades y de acuerdo a las posibilidades reales de recursos, procurando siempre actuar por el bien del paciente y dentro del respeto por la dignidad de la persona.

2. Aplicar triaje para ingreso a UCI. El triaje en este escenario tiene especial relevancia y su aplicación debe ser más estricta. Debe ser aplicado por igual a pacientes que tengan o no Covid-19.

3. Valor de la edad cronológica. La edad per se, como limitante para ingresar a UCI, solo debe ser considerada en última instancia en caso de completa saturación del sistema. 
4. Revalorar pacientes ya admitidos. Maximizar los beneficios es un proceso continuo que se debe tener en cuenta durante toda la internación. La obstinación terapéutica, prohibida por ley, nunca debe ser empleada, resulta especialmente rechazable e inmoral en circunstancias de saturación del sistema.

5. Toma de decisiones por personal experimentado. La responsabilidad en la toma de decisiones debe ser asumida por profesionales de experiencia e idoneidad. Además, debe ser debidamente documentada e informada a la familia.

6. Dar prioridad a los grupos que toman riesgo para asistir. Entre éstos los trabajadores de la salud constituyen una población de riesgo, tanto de contraer una enfermedad aguda (como es el caso de Covid-19), como de padecer los efectos a mediano y largo plazo de una sobrecarga laboral y emocional. Otros grupos también deben ser considerados (policías, por ejemplo) de acuerdo a su función en el contexto sanitario.

7. "Primero llegado, primero servido". Si se presenta la situación en la cual los pacientes no presenten diferencias en la prioridad de ingreso, se recomienda optar por el orden de llegada en base a la experiencia nacional.

8. Considerar los cuidados paliativos. La calidad de vida en los últimos momentos de la vida y una muerte digna es uno de los objetivos básicos de la medicina y parte fundamental del derecho a la salud. El trabajo en conjunto con el equipo de cuidados paliativos mejora la calidad de asistencia y optimiza los cuidados al final de la vida.

9. Procurar siempre un trato humanitario. Implica, entre otros aspectos, permitir que el paciente pueda despedirse de sus seres queridos, y a éstos ayudarlos a realizar el duelo. Las instituciones deben adaptar las condiciones y aplicar protocolos que eviten o disminuyan las posibilidades de contagio, aun en situación de pandemia.

10. Fomentar la participación en la investigación científica en pro de la búsqueda de la mejor evidencia. Una de las pocas certezas que tenemos al momento actual es que no hay evidencia con la calidad suficiente que avale la efectividad de un tratamiento específico, por lo cual todos los tratamientos que se empleen con este objetivo deben ser considerados como de índole compasiva. El uso de estos tratamientos se debe realizar en el contexto de proyectos de investigación aprobados por un comité de ética de la investigación.

\section{c) Triaje en pacientes críticos en condiciones de saturación}

En condiciones de saturación, sugerimos adoptar las características explicitadas en la tabla 2, adaptadas a la realidad de cada centro.

\section{Protección del equipo asistencial}

En este contexto de incertidumbre los equipos asistenciales de Medicina Intensiva ven amenazada su integridad psicofísica ante la eventualidad de la posibilidad de enfermar y ser vectores de la enfermedad para sus seres queridos y a miembros del equipo, carecer de equipos de protección y materiales de trabajo adecuados en calidad y cantidad, estar expuestos a un mayor estrés laboral y moral (debido a la asistencia directa y/o indirecta, posibilidad de tener que asistir a compañeros de trabajo, supervisión de la labor de otros profesionales), enfrentarse a decisiones éticamente complejas, mayor contacto con la muerte que el habitual.

La combinación de estos factores genera ansiedad, angustia, miedo, culpa, lo cual puede afectar el desempeño del o los profesionales, creando un ambiente propicio para el desarrollo de trastornos como el burnout (ver anexo: Continencia y soporte afectivo dirigido a los trabajadores de la salud).

Por lo tanto, las instituciones deben:

- Hacer todo lo posible para disponer de medios suficientes e idóneos en referencia a todos los materiales imprescindibles para la asistencia de este tipo de pacientes: equipos de oxigenoterapia, asistencia ventilatoria mecánica, equipos de protección personal (adecuados a los diferentes grados de exposición/riesgo) en cantidad y calidad suficiente, y con una previsión que permita garantizar un stock adecuado.

- Diseñar sistemas de apoyo psicoafectivo para compensar el desgaste emocional y sus consecuencias.

Las recomendaciones actuales constituyen un marco necesario para mitigar los efectos nocivos de esta crisis (y situaciones similares que probablemente nos depare el futuro) y decidir con base ética mientras se mantiene un cuidado integral del paciente.

\section{Apoyos}

El presente documento cuenta con el apoyo de la Sociedad Uruguaya de Medicina y Cuidados Paliativos.

\section{Summary}

Introduction: the pandemic caused by SARS-CoV2 constitutes a significant challenge for the health system, and especially for Critical Care Units, so we need to 
prepare in many aspects. Likewise, we need to consider there could be an extraordinary demand for beds in critical care units, what would lead to an imbalance between clinical needs and the effective availability of health resources.

Objectives: the study aims to perform a bioethical analysis that could provide guidelines for the assistance of patients in critical care.

Specific objectives: 1) to analyse the main bioethical principles in this context, 2) to support clinicians in the making of difficult decisions, 3) to make the resource allocation criteria specific, 4) to define action lines upon a potential "health's disastrous" scenario

Method: the Uruguayan Society of Intensive Care has generated a space for collective work based on discussion processes. Documents include a bibliographic review and the existing protocols.

Results: the study presents a theoretical analysis that is backed up by the bioethical principles involved in the pandemic context on the scenarios of demand for assistance and, by the arguments calling for a change in the ethical criteria upon the saturation of the health system.

Conclusion: practical recommendations are made: 1) for the making of decisions about admission and discharge in a controlled demand. 2) to define action criteria upon an increase in demand, clearly defining the different scenarios, 3) to apply upon the saturation of the health system.

\section{Resumo}

Introdução: a pandemia causada pelo SARS-CoV2 gera um importante desafio para o sistema de saúde e principalmente para a Medicina Intensiva. É preciso se preparar em vários aspectos. Além disso, considera plausível uma demanda extraordinária por leitos críticos, que pode levar a um desequilíbrio entre as necessidades clínicas e a disponibilidade efetiva de recursos de saúde.

Objetivos: realizar uma análise bioética para orientar o cuidado ao paciente crítico. Objetivos específicos: 1) Analisar os princípios bioéticos fundamentais neste contexto, 2) Apoiar os médicos na tomada de decisões difíceis, 3) Tornar explícitos os critérios de alocação de recursos, 4) Definir linhas de ação perante um possível cenário de " desastre de saúde ".

Métodos: a SUMI gerou um ambiente de trabalho coletivo cujo método de trabalho era deliberativo. A documentação usa a revisão da literatura e os protocolos existentes.

Resultados: o trabalho propõe uma análise teórica documentada sobre os princípios bioéticos envolvidos no contexto da Pandemia, sobre os cenários da demanda de saúde e sobre os fundamentos para uma mudança de critérios éticos em um cenário de saturação do sistema.

Conclusão: são propostas recomendações práticas para: 1) tomada de decisão para admissão e alta sob demanda controlada. 2) critérios de atuação frente ao aumento da demanda, estabelecendo definições dos diferentes cenários. 3) recomendações a serem aplicadas em um cenário de saturação do sistema.

\section{Bibliografía}

1. Ley 18.211. Sistema Nacional Integrado de Salud: normativa referente a su creación, funcionamiento y financiación. Montevideo, 13 de diciembre de 2007. Disponible en: https://legislativo.parlamento.gub.uy/temporales/leytemp7659440.htm. [Consulta: 22/05/2020].

2. Ley 18.335. Pacientes y usuarios de los servicios de salud: se establecen sus derechos y obligaciones. Montevideo, 26 de agosto de 2008. Disponible en: http://www.bps.gub.uy/ bps/file/8261/1/ley_18335_-_pacientes_y_usuarios_de_los_servicios_de_salud.pdf. [Consulta:22/05/2020].

3. Beauchamp T, Childress J. Principios de ética biomédica. Barcelona: Masson, 1999.

4. Pellegrino E. Moral choice, the good of the patient, and the patient's good. En: Moskop J,Kopelman L, eds. Ethics and critical care medicine. Philosophy and Medicine, vol 19. Basel: Springer Nature, 1985:117-38.

5. Gracia D. De la bioética clínica a la bioética global: treinta años de evolución. Acta Bioeth 2002; 8(1):27-39.

6. American Medical Association. Code of medical ethics.Disponible en: https://www.ama-assn.org/delivering- care/ethics/code-medical-ethics-overview [Consulta: 22/05/2020].

7. Beca JP. Problemas éticos en situaciones de catástrofes. Santiago de Chile: Centro de Bioética de la Facultad de Medicina de la Universidad del Desarrollo, 2010. Disponible en: https://medicina.udd.cl/centro-bioetica/files/2010/10/parral.pdf. [Consulta: 22/05/2020].

8. Sociedad Española de Medicina Intensiva, Crítica y Unidades Coronarias. Grupo de Trabajo de Bioética. Recomendaciones éticas para la toma de decisiones en la situación excepcional de crisis por pandemia Covid-19 en las unidades de cuidados intensivos. Madrid: SEMICYUC, 2020. Disponible en: https://semicyuc.org/wp-content/uploads/2020/03/\%C3 \%89tica_SEMICYUC-COVID-19.pdf. [Consulta:22/05/ 2020].

9. Gómez-Batiste X, Martínez-Muñoz M, Blay C, Amblàs J, Vila L, Costa X, et al. Recomendaciones para la atención integral e integrada de personas con enfermedades o condiciones crónicas avanzadas y pronóstico de vida limitado en servicios de salud y sociales: NECPAL-CCOMICOC 3.0 (2016). Barcelona: Institut Català d' Oncologia, 2016. Disponible en: http://ico.gencat.cat/web/.content/minisite/ico/professionals/documents/qualy/arxius/NECPAL-3.0ESPANOL_completo.pdf. [Consulta: 22/05/2020].

10. Rockwood K, Song X, MacKnight C, Bergman H, Hogan D, McDowell I, et al. A global clinical measure of fitness and 
frailty in elderly people. CMAJ 2005;173(5):489-95. doi: 10.1503/cmaj.050051

11. National Institute for Health and Care Excellence. COVID-19 rapid guidelines: critical care. London: NICE, 2020. Disponible en:www.nice.org.uk/guidance/ng159. [Consulta: 22/05/2020].

12. Duems Noriega O. Valoración de la fragilidad: aspecto fundamental en el paciente crítico. RevEspGeriatrGerontol 2018; 53(6):362-6.

13. Sprung C, Joynt G, Christian M, Truog R, Rello J, Nates J. Adult ICU Triage During the Coronavirus Disease 2019 Pandemic: who will live and who will die? Recommendations to Improve Survival. Crit Care Med 2020;48(8): 1196-202.

14. White D, Lo B. A framework for rationing ventilators and critical care beds during the COVID-19 pandemic. JAMA 2020;323(18):1773-4.

15. Vergano M, Bertolini G, Giannini A, Gristina G, Livigni S, Mistraletti G, et al. Raccomandazioni di ética clínica per l'ammissione a trattamentiintensivi e per la loro sospensione, in condizioni eccezionali di squilibrio tra necessità e risorse disponibili. Roma: Società Italiana di Anestesia Analgesia Rianimazione e Terapia Intensiva, 2020. Disponible en: http://www.siaarti.it/SiteAssets/News/COVID19

$\% 20-\% 20$ documenti $\% 20$ SIAARTI/SIAARTI $\% 20-\% 20 \mathrm{Co}-$ vid19\%20-\%20Raccomandazioni\%20di\%20etica\%20clinica.pdf. [Consulta:22/05/2020].

16. Cluzet O, Berro G. La pandemia de COVID-19: consideraciones éticas y médico-legales. Revisado por la Comisión de Asesoramiento Bioético de la Federación Médica del Interior. Montevideo: FEMI, 2020. Disponible en: https:// c6c493a3-18e5-4bd1-92b4-dc602742389c.filesusr.com/ ugd/222cdb_a6ab0c8820d9480c9fbf0e857e35ced3.pdf. [Consulta: 22/05/2020].

17. Emanuel E, Persad G, Upshur R, Thome B, Parker M, Glickman A, et al. Fair allocation of scarce medical resources in the time of Covid-19. N Engl J Med 2020; 382(21):2049-55.

\section{Contribución de autores}

Álvaro Giordano, Ana Canale, Julio Pontet y Luis Núñez participaron en la concepción, diseño, ejecución, análisis, interpretación de los resultados, redacción y revisión crítica de todo el artículo. Natacha Reyes, Armando Cacciatore y Humberto Correa participaron en el análisis, redacción y revisión crítica del artículo.

Álvaro Giordano, https://orcid.org/0000-0001-5468-7555

Ana Canale, https://orcid.org/0000-0001-5210-8214

Julio Pontet, https://orcid.org/0000-0002-6355-652X

Natacha Reyes, https://orcid.org/0000-0001-6067-198X

Armando Cacciatori, https://orcid.org/0000-0003-0697-5557

Humberto Correa, https://orcid.org/0000-0001-5486-3360

Luis Andrés Núñez, https://orcid.org/0000-0002-0916-1615 


\section{Anexo 1. Rol de cuidados paliativos en la asistencia a pacientes críticos}

Los pacientes con enfermedades crónicas avanzadas o aquellos que dado su mal pronóstico no se beneficien de ingresar a UCI, pero se encuentren con una carga sintomática grave, requerirán de una atención que alivie el sufrimiento con un adecuado control sintomático o acompañamiento de fin de vida, asegurando una muerte digna cuando ésta sea ineludible ${ }^{(1,3)}$.

Es en este momento cuando los cuidados paliativos (CP) adquieren un rol preponderante.

Los CP son la asistencia activa, holística, de personas de todas las edades con sufrimiento severo relacionado con la salud debido a una enfermedad grave, y especialmente de quienes están cerca del final de la vida ${ }^{(4)}$.

Su objetivo es mejorar la calidad de vida de los pacientes, sus familias y sus cuidadores.

Incluyen:

- Prevención, identificación precoz, evaluación integral y control de problemas físicos, incluyendo dolor y otros síntomas angustiantes, sufrimiento psicológico, sufrimiento espiritual y necesidades sociales.

- Apoyo a los pacientes para ayudarlos a vivir lo mejor posible hasta la muerte, facilitando la comunicación efectiva, ayudándoles a ellos y a sus familias a determinar los objetivos de la asistencia.

- Se aplican durante el transcurso de la enfermedad, de acuerdo con las necesidades del paciente, en todos los ambientes de atención médica y en todos los niveles.

- Se proporcionan conjuntamente con tratamientos que modifican la enfermedad, siempre que sea necesario.

- Pueden influir positivamente en el curso de la enfermedad.

- No aceleran ni posponen la muerte, afirman la vida y reconocen la muerte como un proceso natural.

- Brindan apoyo a la familia durante el duelo.

- Se proveen reconociendo y respetando los valores y las creencias culturales del paciente y de la familia.

- Los pacientes que se asisten tienen altos niveles sufrimiento por la enfermedad y por las características específicas del momento, miedo y soledad, exacerbados por el menor contacto humano, aislamiento social y confinamiento.

\section{Recomendaciones}

En el marco de una buena comunicación y de trabajo en equipo, y siempre respetando las normas de seguridad y protección del equipo sanitario y del binomio pacien- te-familia, se recomienda realizar los máximos esfuerzos para:

- Brindar una atención humanizada, cercana, individualizada e integral, con el apoyo emocional, espiritual y social necesario para el paciente, su familia y el equipo asistencial.

- Optimizar la asignación de recursos de acuerdo al criterio de Equidad (mayores recursos a pacientes más complejos).

- Facilitar dentro de las posibilidades y protocolos institucionales el acompañamiento por las diferentes vías (virtual, presencial), sobre todo en la etapa de fin de la vida.

- Brindar información por los medios necesarios acerca del estado de salud al propio paciente y sus familiares.

- Identificar personal entrenado en control sintomático o en su defecto capacitar en el uso de pautas de rápido acceso.

- Administrar los recursos materiales y humanos para poder utilizarlos de la forma más efectiva posible.

\section{Control sintomático}

Debe ser individualizado teniendo en cuenta los síntomas derivados de las patologías crónicas preexistentes.

Los síntomas más frecuentes en paciente con infección Covid-19 son el delirium, la disnea y la fiebre persistente $^{(5,6)}$.

El delirium es el síntoma más frecuente, principalmente el que se acompaña de agitación psicomotriz, y constituye la principal causa de sufrimiento y de indicación de sedación paliativa frente a la refractariedad del mismo. Traduce mal pronóstico y rápida evolución al fallecimiento.

Se debe propiciar un ambiente tranquilo, facilitar reorientación y retirar fármacos que puedan agravarlo.

En el caso de la disnea a ningún paciente se le puede negar el acceso al tratamiento con oxigenoterapia (cánula nasal, máscaras con reservorio), en algunos casos se puede plantear el apoyo con OAF, VNI (si están disponibles).

Se debe garantizar el acceso al tratamiento farmacológico para estos síntomas.

La sedación paliativa ${ }^{(7)}$ se plantea cuando el o los síntomas son refractarios a los tratamientos disponibles con el objetivo de evitar un sufrimiento intenso en pacientes cuya muerte se prevé próxima y con su consentimiento explícito, implícito o delegado. 
Es una indicación médica que tiene por objetivo la disminución deliberada del nivel de conciencia del enfermo mediante la administración de los fármacos apropiados (en dosis y combinaciones requeridas, tanto como sea necesario). Se debe iniciar sin dilaciones para lograr un rápido alivio del sufrimiento del enfermo.

Cuando está bien indicada, constituye una buena práctica médica y es ética y deontológicamente obligatoria. No debe instaurase para aliviar la pena de los familiares o la carga laboral, o la angustia de las personas que asisten al enfermo.

Deben cumplirse ciertos requisitos para su implementación:

- presencia de síntomas refractarios,

- evolución a la muerte inminente,

- consentimiento informado, escrito, verbal o delegado a la familia,

- discutida y decidida por el equipo tratante,

- uso de dosis adecuadas y proporcionales al síntoma que se quiere tratar,

- monitorización de la respuesta del paciente (por ejemplo, escala de Ramsay, RASS),

- registro en historia clínica de todo el proceso,

- enmarcarse dentro de los conceptos bioéticos y deontológicos vigentes.

Se debe propiciar un ambiente tranquilo, mantener las medidas de higiene y confort, y retirar monitoreo y alarmas innecesarias.

No olvidar la asistencia y el apoyo al proceso de duelo con prevención del duelo patológico en un momento donde por todas las características analizadas se propicia su aparición.

Se deben realizar los máximos esfuerzos para facilitar las despedidas.

\section{Bibliografía}

1. Sociedad Española de Medicina Intensiva, Crítica y Unidades Coronarias. Grupo de Trabajo de Bioética. Recomendaciones éticas para la toma de decisiones en la situación excepcional de crisis por pandemia Covid-19 en las unidades de cuidados intensivos. Madrid: SEMICYUC, 2020. Dispo- nible en: https://semicyuc.org/wp-content/uploads/2020/ 03/\%C3\%89tica_SEMICYUC-COVID-19.pdf. [Consulta:22/05/2020].

2. Recomendaciones Intersocietarias para la asignación de recursos basados en guías éticas, atención paliativa y criterios de ingreso/egreso en cuidados críticos durante la Pandemia Covid 19. Argentina, Abril de 2020. Disponible en: https://www.bioeticacs.org/iceb/seleccion_temas/pandemia/00108.pdf. [Consulta:18/05/2020].

3. Sociedad Española de Cuidados Paliativos. Orientaciones sobre el control sintomático de enfermos graves afectados por la enfermedad Covid 19 y que requieran atención paliativa o se encuentren próximos al final de la vida. Madrid: SECPAL, 2020. Disponible en: http://www.secpal.com//Documentos/Blog/2020_03_23\%20FIN\%20DE\%20VIDA \%20Y\%20 COVID\%2019\%20_1.\%20Documento\%20para\%20profesionales_1.pdf. [Consulta:22/05/2020].

4. International Association for Hospice And Palliative Care. Palliative care definition. Houston, TX: IAHPC, 2018. Disponible en: https://hospicecare.com/what-we-do/projects/consensus-based-definition-of-palliative-care/definition/. [Consulta: 15/05/2020].

5. Clínica Universidad de Navarra. Servicio de Medicina Paliativa. Guías rápidas de apoyo y control sintomático en pacientes avanzados con COVID19: versión 2.0 (22.03.2020). Navarra: Clínica Universidad de Navarra, 2020. Disponible en: https://cuidadospaliativos.org/ blog/wp-content/uploads/2020/03/Guia-COVID-19.V.2.0_ 22.3.20.pdf. [Consulta: 15/05/2020].

6. Instituto Pallium Latinoamérica Medicina Paliativa. Universidad de Buenos Aires. Instituto de Investigaciones Médicas Alfredo Lanari. Recomendaciones de atención a pacientes graves o últimos días de vida en la crisis del Covid-19. Buenos Aires: Instituto Pallium Latinoamérica Medicina Paliativa,2020.Disponible en: https://www.sc.edu/ study/colleges_schools/socialwork/documents/corona_covid_language_guide_spanish.pdf. [Consulta: 20/12/2020].

7. Píriz Álvarez G, adap. Cuidados paliativos: control de síntomas. Revisión y adaptación a Uruguay de la Dra. Gabriela Píriz Álvarez. Montevideo: MSP, 2014. Disponible en: https://www.gub.uy/ministerio-salud-publica/sites/ministerio-salud-publica/files/documentos/publicaciones/Libro\%20CONTROL\%20DE\%20SINTOMAS\%20EN\%20C UIDADOS\%20PALIATIVOS\%20MINISTERIO\%20DE\% 20SALUD.pdf. [Consulta: 15/05/2020]. 


\section{Anexo 2. Continencia y soporte afectivo dirigido a los trabajadores de la salud}

¿Quiénes y cómo debemos cuidar a los encargados de proteger nuestra salud?

El personal sanitario que trabaja en UCI está sometido a una presión física y psicológica elevadas. Tiene que tomar decisiones clínicas rápidas y trascendentes, y considerarlo insensible frente al sufrimiento de pacientes y familiares, y frente a la muerte de los enfermos (aunque exista un grado de adaptación fruto del trabajo repetido en estas condiciones), es un grave error. El intensivista no puede escapar al efecto que tienen el contacto permanente con la gravedad, el sufrimiento y la muerte ${ }^{(1)}$.

Esto no constituye un fenómeno nuevo, sino que viene acompañando a la dinámica de las UCI desde mucho tiempo atrás. Con el transcurso de los años, y a medida que fueron en aumento las exigencias físicas y psicológicas de este personal, comenzaron a evidenciarse conductas "particulares" de sus integrantes, tanto médicos como enfermeros. Es entonces que hace irrupción el síndrome de Burnout en el entorno de las áreas destinadas a la asistencia de pacientes críticos y también en otras áreas asistenciales.

Con los años, fueron agregándose otros elementos que complejizaron aún más el problema, como la conducta disruptiva. Este tipo de conducta constituye una amenaza para la calidad de la atención y seguridad del paciente, distorsionando la comunicación ${ }^{(2)}$.

El sociólogo Robert Zussman se refiere a la UCI como “...ocupada por pacientes y staff aterrorizados, consumiendo recursos hospitalarios exagerados y tratando pacientes en mucho de los cuales la muerte es el final esperado, la UCI es el laboratorio ideal para explorar la ética médica actual...,"(3).

La pandemia de Covid-19 es algo intangible, desconocido para la sociedad, con un comportamiento impredecible y bizarro. A través de los medios circula información de todo tipo, desde el origen del virus hasta sus características y tratamiento, sumando muchas incertidumbres y pocas certezas.

En nuestra sociedad está presente el temor fundado a la llegada de bajas temperaturas, lo cual aumentaría el número de contagios y por ende de casos graves, con la eventual saturación del sistema.

Además de todo lo expuesto, se agrega otra variable terrible en el contexto de la crisis: la vivencia de la angustia experimentada en cada uno de manera diferente, que esconde el miedo a la muerte ${ }^{(4)}$.

Los trabajadores de la salud son población de riesgo, tanto para contraer la enfermedad infecto-contagiosa, como del padecimiento a mediano y largo plazo de una sobrecarga laboral y emocional.
El número de médicos intensivistas en nuestro país es finito, sabiendo que existe un número de ellos con edades cercanas a los 65 años, situación que favorece las formas más graves de la enfermedad, debido a la asociación de comorbilidades. Resulta lógico asumir que dichos profesionales, a pesar de contar con la fortaleza otorgada por su mayor experiencia, no escaparán al temor, incertidumbre y desgaste físico-emocional al momento de enfrentar a los enfermos con Covid- 19. Sin embargo, esto afectará también a los médicos más jóvenes y al personal de enfermería, por lo tanto, es justo plantear la atención a la situación de estrés moral de los profesionales, aplicando medidas para prevenir y tratar el síndrome de desgaste profesional (burnout) de los profesionales ${ }^{(5)}$.

Entendemos que se deben generar posibles acciones preventivas y correctivas que permitan disminuir las tensiones generadas en el ámbito laboral, permitiendo el mejor relacionamiento entre los integrantes del equipo y, por ende, asegurar la calidad asistencial de las UCI.

Para ello, realizamos las siguientes recomendaciones:

- Procurar siempre una adecuada comunicación. Cuando la misma se emite en una forma precisa, clara y empática, las relaciones interpersonales y entre los equipos se tornan más armoniosas ${ }^{(6)}$.

- Generación de reuniones grupales entre los integrantes del equipo de salud. Instancia en la cual se puedan expresar situaciones de tensión y estrés emocional vividas durante la asistencia. Se deberán analizar y lograr la generación de actitudes que propicien acciones de mejora. Estas reuniones, creemos, que facilitarán la descompresión tensional y constituirán una importante herramienta para combatir el burnout y las conductas disruptivas. Permitirían también potenciar al equipo asistencial desarrollando técnicas de comunicación y abrir líneas de investigación que aseguren que "lo que hacemos, lo hacemos bien" ${ }^{\text {(7) }}$.

- Rotación del personal de salud de forma equitativa. Siempre que la situación lo permita, se debe procurar esta rotación en las áreas de asistencia a pacientes con Covid-19 con la finalidad de proteger al personal no sólo del contagio (vinculado a la exposición de la mayor carga viral), sino también de la tensión física y emotiva continua. Una sugerencia podría ser la rotación semanal dentro del área Covid-19.

- No se debe agotar al capital humano más joven, exponiéndolo con mayor frecuencia a esas áreas, 
aplicando el concepto desacertado de que por su condición etaria tendrá menos chance de adquirir la enfermedad, no siendo éticamente recomendable.

- La creación de una actitud de autocuidado ${ }^{(6)}$ optimizará seguramente nuestras herramientas para enfrentar este desafío de la medicina a nivel mundial.

\section{Bibliografía}

1. Gómez Rubí J, Caparrós Fernández de Aguilar T. El paciente crítico y la medicina intensiva. En: Gómez Rubí J, Abizanda Campos R. Bioética y medicina Intensiva: dilemas éticos en el paciente crítico. Barcelona: EdikaMed, 1998:17-28.

2. Godino M, Barbato M, Ramos L, Otero M, Briozzo L. Encuesta nacional de comportamientos disruptivos en el equipo de salud. Rev Méd Urug 2014; 30(4):235-46.

3. Zussman R. Intensive care: medical ethics and the medical profession. Chicago: University of Chicago Press, 1992.

4. Vallejo Nagera J. Reacción vivencial anormal. Psiquiatría clínica. En: Vallejo Nagera J. Introducción a la Psiquiatría. Madrid: Editorial Científico-Médica, 1979:121-42.
5. Sociedad Española de Medicina Intensiva, Crítica y Unidades Coronarias. Grupo de Trabajo de Bioética. Recomendaciones éticas para la toma de decisiones en la situación excepcional de crisis por pandemia Covid-19 en las unidades de cuidados intensivos. Madrid: SEMICYUC, 2020. Disponible en: https://semicyuc.org/wp-content/uploads/2020/03/\% C3\%89tica_SEMICYUC-COVID-19.pdf. [Consulta: 28/04/ 2020].

6. Yordi M, Díaz M. Cuidando la salud mental del personal de enfermería en la pandemia Covid-19 (Videoconferencia $\mathrm{N}^{\circ}$ 16). Ciclo de videoconferencias en el marco del Plan de Capacitación de la Comisión Nacional de Enfermería del MSP. Disponible en https://www.youtube.com/watch? $\mathrm{v}=\mathrm{gH}-$ EJHau9Iv0\&list=PL4O9exeLSKwyYdzgTiwX9SCYWtvdiqv7n\&index=16. [Consulta: 22/12/2020].

7. González Garrido C, Martínez Rodríguez S. La enfermería en la UCI. En: Gómez Rubí J, Abizanda Campos R. Bioética y medicina Intensiva:dilemas éticos en el paciente crítico. Barcelona: EdikaMed, 1998:51-63. 


\section{Anexo 3. Escalas de apoyo en la valoración de pacientes}

Tabla A. Instrumento NECPAL CCOMS-ICO@ versión 3.02016 ESP.

\begin{tabular}{|c|c|c|c|}
\hline Pregunta sorpresa (a / entre profesionales) & \multicolumn{2}{|c|}{ ¿Le sorprendería que este paciente muriese a lo largo del próximo año? } & + \\
\hline \multirow[t]{2}{*}{ "Demanda" o "Necesidad" } & \multicolumn{2}{|c|}{$\begin{array}{l}\text { - Demanda: ¿Ha habido alguna expresión implicita o explícita de } \\
\text { limitación de esfuerzo terapéutico o demanda de atención paliativa de } \\
\text { paciente, familia, o miembros del equipo? }\end{array}$} & \multirow[t]{2}{*}{+} \\
\hline & \multicolumn{2}{|c|}{ - Necesidad: identificada por profesionales miembros del equipo } & \\
\hline \begin{tabular}{|l|} 
Indicadores clínicos generales: \\
6 meses
\end{tabular} & - Declive nutricional & - Pérdida peso $>10 \%$ & + \\
\hline $\begin{array}{l}\text { - Severos, sostenidos, progresivos, no } \\
\text { relacionados proceso intercurrente reciente }\end{array}$ & \multirow[t]{2}{*}{ - Declive funcional } & - Deterioro Karnofsky o Barthel > 30\% & \multirow[t]{2}{*}{+} \\
\hline \multirow{2}{*}{ - Combinar severidad CON progresión } & & - Pérdida de >2 ABVDs & \\
\hline & - Declive cognitivo & - Deterioro Minimental/Pfeiffer & \\
\hline Dependencia severa & \multicolumn{2}{|c|}{- Karnofsky $<50$ o Barthel $<20$} & + \\
\hline \multirow{3}{*}{ Síndromes geriátricos } & $\begin{array}{l}\text { - Caídas - Úlceras por } \\
\text { presión }\end{array}$ & - Datos clínicos anamnesis & \\
\hline & - Disfagia - Delirium & - repetidos $>2$ & \\
\hline & - Infecciones a & - o persistentes & \\
\hline Síntomas persistentes & $\begin{array}{l}\text { Dolor, debilidad, } \\
\text { anorexia, disnea, } \\
\text { digestivos... } \\
\end{array}$ & - Checklist sintomas (ESAS) & + \\
\hline \multirow{2}{*}{ Aspectos psicosociales } & $\begin{array}{l}\text { Distrés y } / 0 \text { trastorno } \\
\text { adaptativo severo }\end{array}$ & - Detección de malestar emocional $(\mathrm{DME})>9$ & \\
\hline & $\begin{array}{l}\text { Vulnerabilidad social } \\
\text { severa }\end{array}$ & - Valoración social y familiar & \\
\hline Multimorbilidad & \begin{tabular}{|l|}
$>2$ enfermedades \\
crónicas (de lista de \\
indicadores específicos)
\end{tabular} & - Test Charlsson & Si/no \\
\hline & \multirow{2}{*}{$\begin{array}{l}\text { Valoración de la } \\
\text { demanda o intensidad } \\
\text { intervenciones }\end{array}$} & $\begin{array}{l}->2 \text { ingresos urgentes o no planificados } 6 \\
\text { meses }\end{array}$ & \multirow[b]{2}{*}{+} \\
\hline Uso de recursos & & $\begin{array}{l}\text { Aumento demanda o intensidad de } \\
\text { intervenciones (atención domiciliaria, } \\
\text { intervenciones enfermería, etc) }\end{array}$ & \\
\hline Indicadores específicos & \begin{tabular}{|l|} 
Cáncer, EPOC, ICC, y \\
hepática, y renal, AVC, \\
demencia, \\
neurodegenerativas, \\
SIDA, otras \\
enfermedades \\
avanzadas
\end{tabular} & - Para ser desarrollados como anexos & + \\
\hline
\end{tabular}


Tabla B. Escala de fragilidad clínica de Rockwood

\begin{tabular}{|c|c|}
\hline Categoría & Características \\
\hline 1 & $\begin{array}{l}\text { En muy buena forma. Personas que están fuertes, activas, vigorosas y } \\
\text { motivadas. Son personas que suelen practicar ejercicio con regularidad. Son } \\
\text { de los que están en mejor forma para su edad. }\end{array}$ \\
\hline 2 & $\begin{array}{l}\text { En forma. Personas sin sintomas de enfermedad activa, pero que están menos } \\
\text { en forma que las de la categoria } 1 \text {. Suele ocurrir que se ejercitan o están muy } \\
\text { activas por temporadas, por ejemplo, según la estación. }\end{array}$ \\
\hline 3 & $\begin{array}{l}\text { En buen estado. Personas que tienen bien controlados sus problemas } \\
\text { médicos, pero que no llevan actividad fisica regular más allá de los paseos } \\
\text { habituales. }\end{array}$ \\
\hline 4 & $\begin{array}{l}\text { Vulnerables. Aunque no dependen de otros que les ayuden en la vida diaria, a } \\
\text { menudo los sintomas limitan sus actividades. Suelen quejarse de estar «lentos» } \\
\text { o cansados durante el dia. }\end{array}$ \\
\hline 5 & $\begin{array}{l}\text { Levemente frágiles. Estas personas a menudo tienen un enlentecimiento más } \\
\text { evidente y necesitan ayuda para las actividades de la vida diaria importantes } \\
\text { (economia, transporte, labores domésticas, medicación). Es tipico que la } \\
\text { fragilidad leve vaya dificultando salir solos de compras o a pasear y hacer la } \\
\text { comida o las tareas del hogar. }\end{array}$ \\
\hline 6 & $\begin{array}{l}\text { Moderadamente frágiles. Personas que necesitan ayuda para todas las } \\
\text { actividades en el exterior y para realizar las tareas domésticas. En casa, suelen } \\
\text { tener problemas con las escaleras y necesitan ayuda con el baño, y pueden } \\
\text { requerir alguna asistencia para vestirse (guía y acompañamiento). }\end{array}$ \\
\hline 7 & $\begin{array}{l}\text { Fragilidad grave. Dependen totalmente para el cuidado personal, sea cual } \\
\text { fuere la causa (fisica o cognitiva). Aun asi, parecen estables y sin riesgo de } \\
\text { muerte (en los siguientes } 6 \text { meses). }\end{array}$ \\
\hline 8 & $\begin{array}{l}\text { Fragilidad muy grave. Totalmente dependientes, se acercan al final de la vida. } \\
\text { Es tipico que ni siquiera se recuperen de afecciones menores. }\end{array}$ \\
\hline 9 & $\begin{array}{l}\text { Enfermo terminal. Se aproximan al final de la vida. Esta categoría se aplica a } \\
\text { personas con esperanza de vida }<6 \text { meses y sin otros signos de fragilidad. }\end{array}$ \\
\hline \multicolumn{2}{|c|}{$\begin{array}{l}\text { En personas con demencia, el grado de fragilidad se corresponde con el grado de } \\
\text { demencia. Son sintomas comunes de demencia leve olvidar los detalles de un } \\
\text { acontecimiento reciente aun recordando el evento en si, la repetición de una misma pregunta } \\
\text { o relato y el aislamiento social. }\end{array}$} \\
\hline \multicolumn{2}{|c|}{$\begin{array}{l}\text { En la demencia moderada, la memoria reciente está muy afectada, aunque parezca que } \\
\text { recuerdan bien los acontecimientos de su pasado. Con pautas, pueden cuidarse solos. }\end{array}$} \\
\hline
\end{tabular}

Tabla C. Escala funcional de Karnofsky

\begin{tabular}{|l|c|}
\hline \multicolumn{1}{|c|}{ Escala funcional de Karnofsky } & \\
\hline \multicolumn{1}{|c|}{ Caracteristica } & Puntaje \\
\hline Asintomático, sin evidencia de enfermedad. & 100 \\
\hline Capaz de realizar actividad normal. Signos o sintomas menores de enfermedad. & 90 \\
\hline Actividad normal con effuerzo. Algunos sintomas o signos de enfermedad. & 80 \\
\hline Incapaz de realizar actividad normal o trabajar. Se vale por si mismo. & 70 \\
\hline Asistencia ocasional. Se hace cargo de la mayoría de sus necesidades. & 60 \\
\hline Considerable asistencia. Frecuentes cuidados médicos. & 50 \\
\hline Imposibilitado. Requiere cuidados especiales y asistencia. & 40 \\
\hline Gravemente imposibilitado. La hospitalización está indicada, aunque la muerte no es inminente. & 30 \\
\hline Muy enfermo. Precisa hospitalización. Requiere tratamiento de soporte activo. & 20 \\
\hline Moribundo. & 10 \\
\hline Exitus. & 0 \\
\hline
\end{tabular}

